\title{
The Protective Effects of Statins towards Vessel Wall Injury Caused by a Stent Retrieving Mechanical Throm- bectomy Device : A Histological Analysis of the Rabbit Carotid Artery Model
}

\author{
Seung Hwan Lee, ${ }^{1}$ Hee Sup Shin, ${ }^{1}$ Inho $\mathrm{Oh}^{2}$ \\ Department of Neurosurgery, Kyung Hee University Hospital at Gangdong, College of Medicine, Kyung Hee University, Seoul, Korea \\ Department of Neurosurgery, ${ }^{2}$ Veterans Health Service Medical Center, Seoul, Korea
}

Objective : Endovascular mechanical thrombectomy (MT) has been regarded as one of the standard treatments for acute ischemic stroke caused by large vessel occlusion. Despite the wide use of stent retrievers for MT, arterial intimal damage caused when deployed stent is pulled has been a certain disadvantage. We hypothesized that statin could protect and stabilize vessel damage after endovascular MT using a stent retriever. In this animal study, we observed the protective effects of the statins towards MTinduced vessel wall injury.

Methods : Twenty-eight carotid arteries of fourteen rabbits were used in the experiments with MT using stent retriever. We divided the rabbits into four groups as follows : group 1, negative control; group 2, positive control; group 3, statin before MT; and group 4, statin after MT. After MT procedures, we harvested the carotid arteries and performed histomorphological and immunohistochemical analyses.

Results : In histomorphological analysis with hematoxylin and eosin and Masson's trichrome stain, significant intimal thickening $(p<0.05)$ was observed in the positive control (group 2), compared to in the negative control (group 1). Intimal thickening was improved in the statin-administered groups (groups 3 and 4 vs. group $2, p<0.05$ ). We also observed that statin administration after MT (group 4) resulted in a more effective decrease in intimal thickness than statin administration before MT (group 3) ( $p<0.05$ ). We performed immunohistochemical analysis with the antibodies for tumor necrosis factor-alpha (TNF- $\alpha$ ), cluster of differentiation $(C D) 11 b$, and CD163. In contrast to the negative control (group 1), the stained percentage areas of all immunological markers were markedly increased in the positive control (group 2) $(p<0.05)$. Based on statin administration, the percentage area of TNF- $\alpha$ staining was significantly reduced $(p<0.05)$ in group 3 , compared to the positive control group (group 2). However, significant differences were not observed for CD11b and CD163 staining. In group 4, no significant differences were observed for TNF- $\alpha$, CD11b, and CD163 staining $(p \geq 0.05)$. The differences in the percentage areas of the different markers between the statin-administered groups (groups 3 and 4) were also not revealed.

Conclusion : We presented that statin administration before and after MT exerted protective effects towards vessel wall injury. The efficacy of statins was greater post-administration than pre-administration. Thus, statin administration in routine prescriptions in the peri-procedural period is strongly advised.

Key Words : Thrombectomy · Stents · Hydroxymethylglutaryl-CoA reductase inhibitors · Rabbits.

- Received : October 23, 2020 •Revised : December 11, 2020 •Accepted : March 3, 2021

- Address for reprints : Hee Sup Shin

Department of Neurosurgery, Kyung Hee University Hospital at Gangdong, College of Medicine, Kyung Hee University, 892 Dongnam-ro, Gangdong-gu, Seoul 05278, Korea Tel : +82-2-440-6090, Fax : +82-2-440-8404, E-mail : realeponym@hanmail.net, ORCID : https://orcid.org/0000-0002-5286-8448 


\section{INTRODUCTION}

Intravenous (IV) infusion of recombinant tissue plasminogen activator (rt-PA) is a well-established medical treatment for acute ischemic stroke (AIS) caused by large vessel occlusion. Despite its therapeutic benefits, its recanalization rate remains under $40 \%$, as reported in the previous literature ${ }^{1,5,10,33)}$. Since recanalization is an influential decisive factor of good clinical outcome ${ }^{5)}$, clinicians have continued their efforts in improving its recanalization rate. Since the advent of the Merci device in $2001^{36)}$, endovascular mechanical thrombectomy (MT) has been regarded as one of the standard AIS treatments. In 2015, five randomized controlled trials (RCTs) reported the efficacy and safety of stent retrievers ${ }^{4,8,15,18,34)}$. These reports showed that stent retrievers, compared to IV rt-PA infusion, showed an enhanced clinical outcome and a markedly improved recanalization rate of over $80 \%$. In this context, stent retrievers were suggested as first-line AIS treatments in the American Heart Association/American Stroke Association guidelines for early AIS management ${ }^{32)}$.

Stent retrievers have since been used widely for MT; however, certain disadvantages due to the characteristics of a self-expandable stent have been brought to light. One of them is the arterial intimal damage caused when the deployed stent is pulled in an expanded state. It is a type of direct mechanical injury, which results from friction between the arterial wall and the tightly contacted stent, which was repeatedly pulled to capture the clot. Some clinical analyses and animal studies confirmed the intimal and medial damage that occurs due to direct mechanical irritation by the stent retriever, resulting in vasospasm, intimal denudation, intimal hyperplasia, medial thickening, and inflammatory reaction ${ }^{2,7,13,27,28)}$. These morphological changes that occurred after MT using stent retrievers were reported to cause clinical symptoms due to vessel reocclusion and stenosis in the acute and chronic phases, respectively. Regarding this concern about vessel wall injury, clinicians are attempting to reduce the number of times MT is being performed and protect the arterial wall during the procedure or promote recovery of the injured intima by using vascular protective agents.

Besides the powerful low-density lipoprotein (LDL)-lowering effect, the pleiotropic effect of statins is known to improve endothelial function, enhance atherosclerotic plaque stability, decrease oxidative stress and inflammation, and inhibit the thrombogenic response ${ }^{25)}$. We hypothesized that statins could protect and stabilize vessel damage and reduce the inflammatory reaction that occurred after endovascular MT using a stent retriever. Thus, in this study, we observed the protective effects of statins towards MT-induced vessel wall injury using experimental animal models.

\section{MATERIALS AND METHODS}

Animal experimental procedures were conducted according to the International Guide for the Care and Use of Laboratory Animals, and were approved by the Institutional Animal Care and Use Committee of Kbio Health (Osong Advanced Medical Industry Promotion Foundation, Osong, Korea; Accession number KBIO-IACUC-2019-127). The experimental study was approved by the Animal Welfare Act, and all animals received humane care. The Principles of Laboratory Animal Care formulated by the Institute of Laboratory Animal Resources (National Research Council, NIH Publication No. 85-23, revised 1996) were followed.

\section{Animal models and care}

We chose rabbits as the experimental animal models for the following reasons. First, the diameter (about $3 \mathrm{~mm}$ ) of the rabbit carotid artery is similar to that of the human middle cerebral artery (MCA) of the M1 segment. The commonly occluded cerebral artery in AIS in humans is the MCA, and the stent retriever used in this study was suitable for the human MCA. Although, unlike rabbit carotid artery, human MCA does not have external elastic lamina, we considered the similarity of vessel diameter between rabbit carotid artery and human MCA. Second, the action mechanisms of statins in rabbits resemble those in humans ${ }^{30)}$. Third, the features of histopathologically and immunohistochemically stained tissues of rabbits are similar to those of humans. Fourth, rabbits possess excellent regenerative abilities and the capacity to withstand stress. Fifth, breeding and anesthetic methods for rabbits are easy to perform and well-established. Atorvastatin is a 3-hydorxy-3-methylglutaryl-coenzyme-A (HMG-CoA) reductase inhibitor that is widely used for clinical and animal model applications. In a previous study, the therapeutic and safe dosage of atorvastatin in rabbits was reported to be 3-10 $\mathrm{mg} / \mathrm{kg}^{3)}$. We administered $20 \mathrm{mg}$ atorvastatin to rabbits orally. 
Fourteen male New Zealand white rabbits (12-14 weeks, 2.6-3.0 kg; SamtacoBio, Ansan, Korea) were housed individually in metal cages for 15 days. We fed the rabbits with a regular chow diet and maintained the following cage conditions: a 12-hour light-dark cycle (150-300 Lux [light cycle from 08:00 to $20: 00]$ ), temperature at $23 \pm 3^{\circ} \mathrm{C}$, and air ventilation at $10-20$ times/h. There was no dropout of animals during the study period.

The experimenters, wearing lab gowns that were autoclaved $\left(121^{\circ} \mathrm{C}, 20\right.$ minutes), handled the rabbits by following the Association for Assessment and Accreditation of Laboratory Animal Care International system guidelines.

\section{Experimental groups}

We performed statistical power analysis using $G^{\star}$ Power ver. 3.1 (Heinrich Heine Universität Düsseldorf, Düsseldorf, Germany) to determine the sample size ${ }^{11,12)}$. The total sample size calculated by power analysis was 24 , and we included $28 \mathrm{ca}$ rotid arteries of 14 rabbits. We divided the rabbits into four groups as follows (Table 1) : group 1 (negative control, $\mathrm{n}=4 \mathrm{ca}-$ rotid arteries of 2 rabbits), no MT or statin administration; group 2 (positive control, $\mathrm{n}=8$ carotid arteries of 4 rabbits), MT without statin administration; group 3 (statin before MT, $\mathrm{n}=8$ carotid arteries of 4 rabbits), MT with statin administration for seven days before MT; group 4 (statin after MT, $n=8$ carotid arteries of 4 rabbits), MT with statin administration for seven days after MT.

The experimental and breeding period was 14 days. Group 1 was the negative control group, and MT was not performed for the group 1 rabbits. MT was performed on experimental day 8 for the group 2, group 3, and group 4 rabbits. Atorvastatin was not administered to the negative (group 1) and positive (group 2) control group rabbits. We administered atorvastatin (20 mg) (LipiTOR; Jeil Pharmaceutical Co., Ltd., Seoul,
Korea) daily via a $5 \mathrm{~F}$ oral gavage tube to the group 3 and group 4 rabbits. We maintained atorvastatin administration for seven days before (day 1-7) and after (day 8-14) MT for the group 3 and group 4 rabbits, respectively. On day 15, the rabbits of all groups were euthanized using deep isoflurane (>5\%) (Forane; JW Pharmaceutical, Seoul, Korea) anesthesia, followed by IV potassium chloride injection.

\section{Procedure for endovascular MT}

Rabbits were inducted to general anesthesia by administering an intramuscular tiletamine/zolazepam $(15 \mathrm{mg} / \mathrm{kg}$, Zoletil; Virbac Korea, Seoul, Korea) and xylazine $(5 \mathrm{mg} / \mathrm{kg}$, Rompun; Bayer Korea, Ansan, Korea) injection. We maintained isoflurane $(<3 \%)$ anesthesia and oxygen with a respiratory anesthesia device (Fabius GS premium; Dräger Medical $\mathrm{GmbH}$, Lübeck, Germany). After shaving the groin area of the rabbit, we made a skin incision (length, $2 \mathrm{~cm}$ ) and exposed the right femoral artery by carefully dissecting the muscle and fascia layers (Fig. 1).

MT was performed using the angio machine (Artis Zee Multi-Purpose; Siemens Healthineers AG, Erlangen, Germany). The endovascular approach was achieved via the right femoral artery using a $4 \mathrm{~F}$ sheath. We navigated the microcatheter (Trevo Pro 18; Stryker Neurovascular, Fremont, CA, USA) through the right femoral artery to the distal carotid artery. The stent retriever (Trevo XP Provue $4 \times 20 \mathrm{~mm}$; Stryker Neurovascular, Fremont, CA, USA) was introduced into the microcatheter and deployed in the carotid artery. For tight apposition of the stent to the vessel wall, we used the 'push and fluff technique' while deploying the stent ${ }^{16)}$. After 2-3 minutes, the deployed stent was pulled back to the proximal segment of the carotid artery (Fig. 2). We repeated this procedure for both carotid arteries thrice. Three consecutive MT procedures are considered acceptable in a real clinical situation, and

Table 1. Study schedule and animal groups

\begin{tabular}{|c|c|c|c|c|}
\hline & Day 1-7 & Day 7 & Day 8-14 & Day 15 \\
\hline Group 1 (negative control, $\mathrm{n}=4$ arteries of 2 rabbits) & - & - & - & Euthanized \\
\hline Group 2 (positive control, $n=8$ arteries of 4 rabbits) & - & MT & - & Euthanized \\
\hline Group 3 (statin before MT, n=8 arteries of 4 rabbits) & Atorvastatin 20 mg/day & MT & - & Euthanized \\
\hline Group 4 (statin after MT, $\mathrm{n}=8$ arteries of 4 rabbits) & - & MT & Atorvastatin $20 \mathrm{mg} /$ day & Euthanized \\
\hline
\end{tabular}

Group 1 is the negative control group without mechanical thrombectomy (MT). MT was performed on experimental day 8 for the group 2, group 3, and group 4 rabbits. Group 2 is the positive control group without statin administration. We administered $20 \mathrm{mg}$ atorvastatin daily to the group 3 (7 days before MT, day 1-7) and group 4 (7 days after MT, day 8-14) rabbits. On day 15, the rabbits of all groups were euthanized 

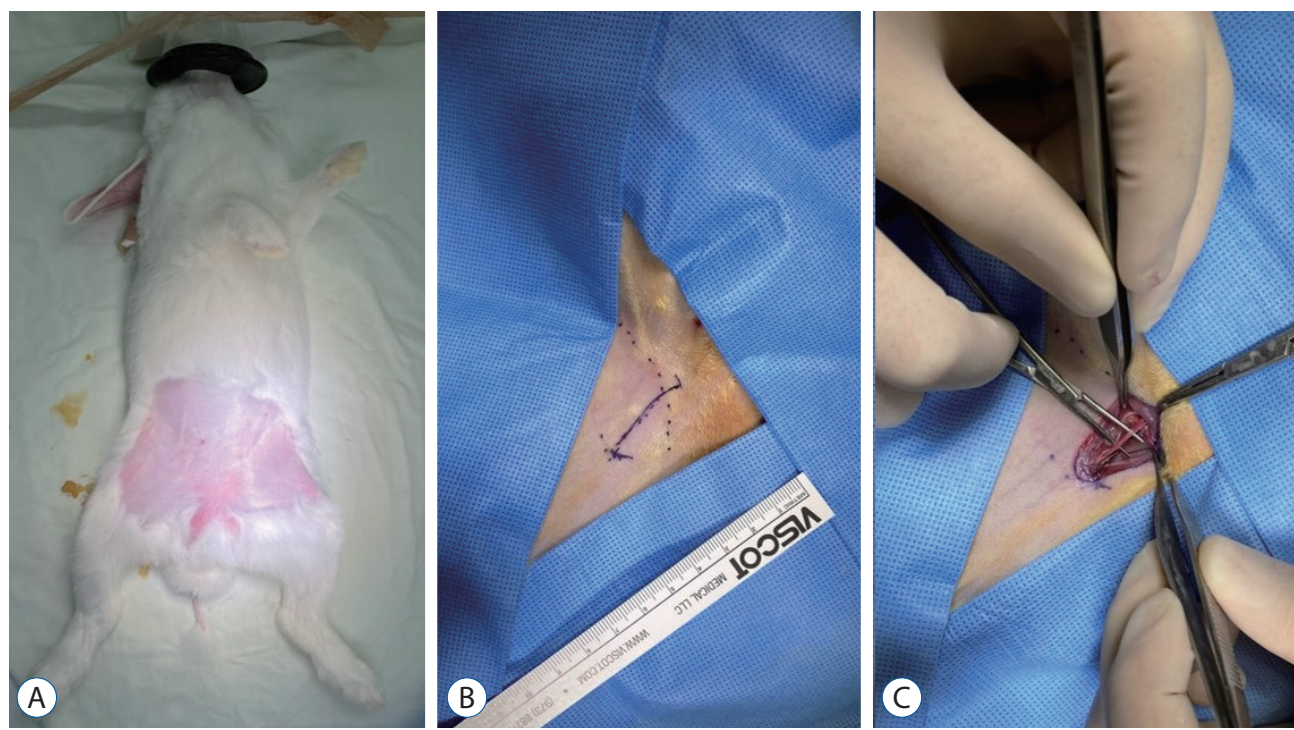

Fig. 1. Exposure of the rabbit right femoral artery. After shaving the groin area of the rabbit (A), we made a skin incision (length, $2 \mathrm{~cm}$ ) (B). The right femoral artery was exposed by carefully dissecting the muscle and fascia layers (C).
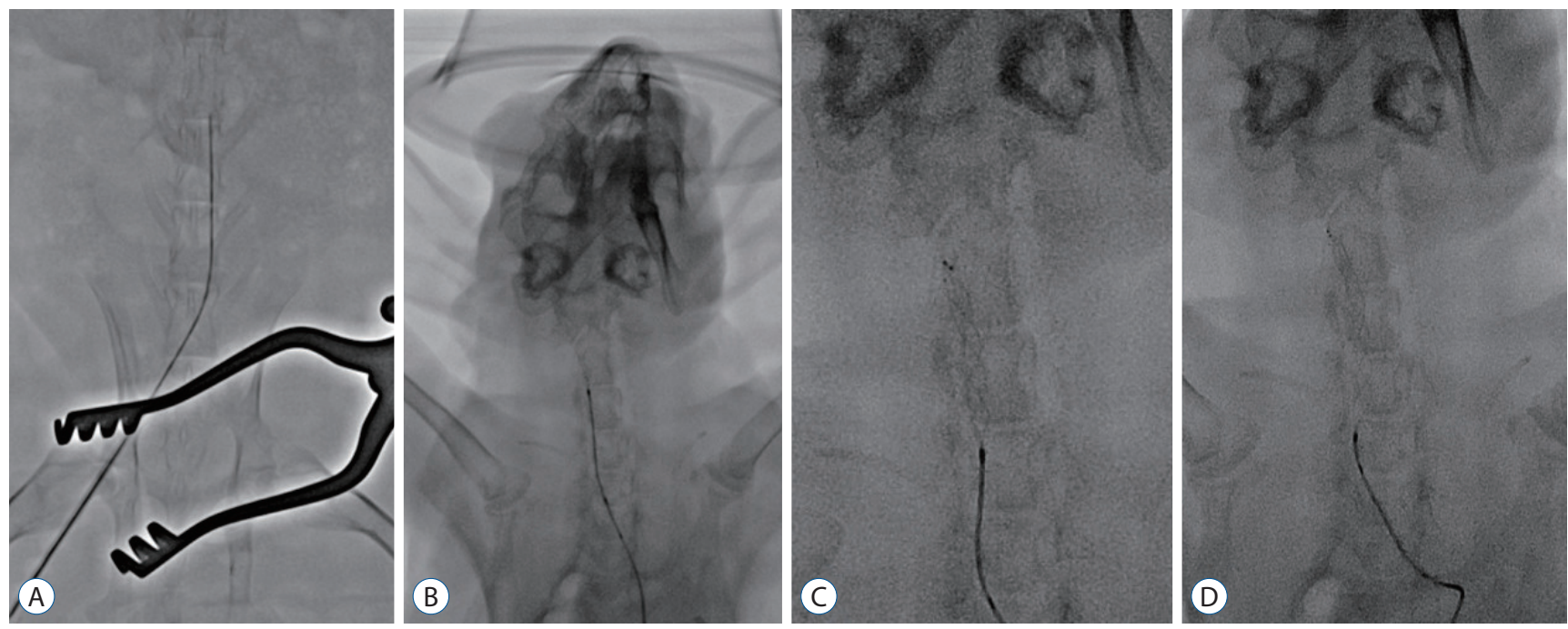

Fig. 2. Procedure for endovascular MT using stent retrievers in the rabbit. The microcatheter was navigated via the right femoral artery to the distal carotid artery through a $4 \mathrm{~F}$ sheath (A). A stent retriever was introduced into the microcatheter and deployed in the carotid artery (B). For tight apposition of the stent to the vessel wall, we used the 'push and fluff technique' while deploying the stent (C). After 2-3 minutes, the deployed stent was pulled back to the proximal segment of the carotid artery (D).

are presumed to cause adequate vessel wall injury.

The microcatheter and femoral artery sheath were subsequently removed after MT, and the right femoral artery was ligated. Muscle and fascia were sutured with absorbable sutures, and the skin was closed with 4-0 nylon suture.

\section{Histological and immunohistochemical analyses}

After euthanizing the rabbits, the carotid arteries were har- vested. We removed both carotid arteries from the proximal to the distal segments. The excised tissues were fixed in $10 \%$ normal buffered formalin for 24 hours and subsequently embedded in paraffin blocks. The tissue cross-section was cut into $4-\mu \mathrm{m}$-thick slices using a microtome and mounted on microscope slides. We stained the slides with hematoxylin and eosin (H\&E) and Masson's trichrome. Additionally, the slides were stained immunohistochemically with antibodies to clus- 
ter of differentiation (CD)163 (ABCAM, Cambridge, MA, USA), CD11b (NovusBio, Littleton, CO, USA), and tumor necrosis factor-alpha (TNF- $\alpha$; NovusBio, Littleton, CO, USA).

We obtained digital images of the slides using a Carl Zeiss Axio Observer 5 microscope and the Zen microscope software (Carl Zeiss AG, Jena, Germany). Histomorphometry was performed using the Image J program (National Institutes of Health, Bethesda, MD, USA). We observed the inner vessel wall mechanical injury by measuring the thickness of the tunica intima (defined as the vessel wall layer from the internal elastic lamina to the lumen) stained with H\&E and Masson's trichrome. We selected the slide with the middle segment of the carotid artery and measured the intima at the thickest position. We also evaluated the percentage areas of the tissue immunohistochemically stained with antibodies to rabbit macrophages, CD163, CD11b, and TNF- $\alpha$, to measure the inflammatory reaction.

\section{Statistical analysis}

Data analysis was conducted using SPSS ver. 23.0 (SPSS Inc., Chicago, IL, USA). The variables were expressed as the mean \pm standard deviation, and were compared using one-way analysis of variance (ANOVA) for three or more groups with posthoc (Duncan's method). $p$-value $<0.05$ was considered statistically significant.

\section{RESULTS}

\section{Histomorphological analysis of the tunica intima}

We measured the thickness of the tunica intima stained with H\&E and Masson's trichrome microscopically (Fig. 3). Significant intimal thickening was observed in group 2, while decreased intimal thickness was observed in the statin-administered groups (groups 3 and 4). We then measured the intimal thickness of the rabbits in each group by histomorphological analysis (Table 2 and Fig. 4). Significant intimal thickening $(p<0.05)$ was observed in the positive control (group 2, 48.04 $\pm 12.28 \mu \mathrm{m}$ of H\&E and $42.72 \pm 8.82 \mu \mathrm{m}$ of Masson's trichrome), compared to in the negative control (group 1, $3.74 \pm 0.62 \mu \mathrm{m}$ of $\mathrm{H} \& \mathrm{E}$ stain and $5.84 \pm 0.63 \mu \mathrm{m}$ of Masson's trichrome). Intimal thickening was improved in the statin-administered groups (groups 3 and 4 vs. group 2, $p<0.05$ ). The measured thickness was $30.88 \pm 10.41 \mu \mathrm{m}$ (H\&E) and $28.36 \pm$
$9.86 \mu \mathrm{m}$ (Masson's trichrome) in group 3 and $15.86 \pm 3.19 \mu \mathrm{m}$ (H\&E) and 14.93 $\pm 5.75 \mu \mathrm{m}$ (Masson's trichrome) in group 4. We also observed that statin administration after MT (group 4) resulted in a more effective decrease in the intimal thickness than statin administration before MT (group 3) $(p<0.05)$.

\section{Immunohistochemical analysis of the statin ef- fect}

Immunohistochemical staining of macrophage markers was performed using TNF- $\alpha$, CD11b, and CD163 to evaluate the anti-inflammatory effect of statins (Fig. 5). The stained percentage areas of all immunological antibodies were markedly increased in group 2 (Table 2 and Fig. 6). In contrast to group 1 (TNF- $\alpha, 10.55 \pm 3.63$ area\%; CD11b, $11.15 \pm 4.95$ area\%; and $\mathrm{CD} 163,14.25 \pm 1.28$ area\%), group 2 showed significantly increased percentage areas of TNF- $\alpha(21.75 \pm 6.78$ area $\%$, $p<0.05)$ and CD11b, (19.64 \pm 5.19 area\%, $p<0.05)$. However, percentage area of CD163 (24.54 \pm 8.59 area\%) did not significantly increased $(p>0.05)$. Although the percentage areas of the markers based on statin administration were decreased, significant differences were distinguished among the markers. In group 3 , the percentage area of TNF- $\alpha$ staining was significantly reduced $(p<0.05)$, but significant differences were not observed for CD11b and CD163 staining compared to group 2. In group 4 , no significant differences were observed for TNF- $\alpha$, CD11b, and CD163 staining $(p \geq 0.05)$ compared to group 2 . The differences in the percentage areas of the different markers between the statin-administered groups (groups 3 and 4) were also not revealed.

\section{DISCUSSION}

\section{The protective effects of statins towards MT- induced vessel wall injury}

We observed the protective effects of statins towards MTinduced vessel wall injury. Intimal thickening after endothelial injury before or after statin administration, compared to that without statin administration, was found to be significantly reduced. To date, this animal experiment performed to elucidate the role of statins in vascular protection is the first report on the reduction of the after-effects of MT-induced endothelial injury. This study demonstrated that group 2 (MT without statin administration) showed increased intimal thickening, 
$H \& E$

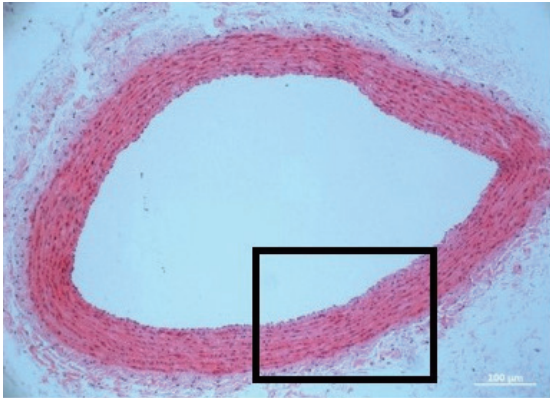

G2

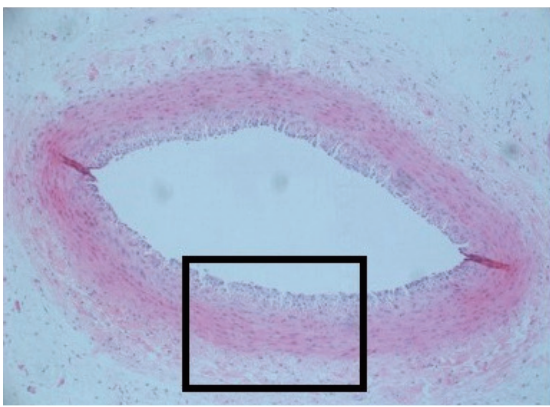

G3
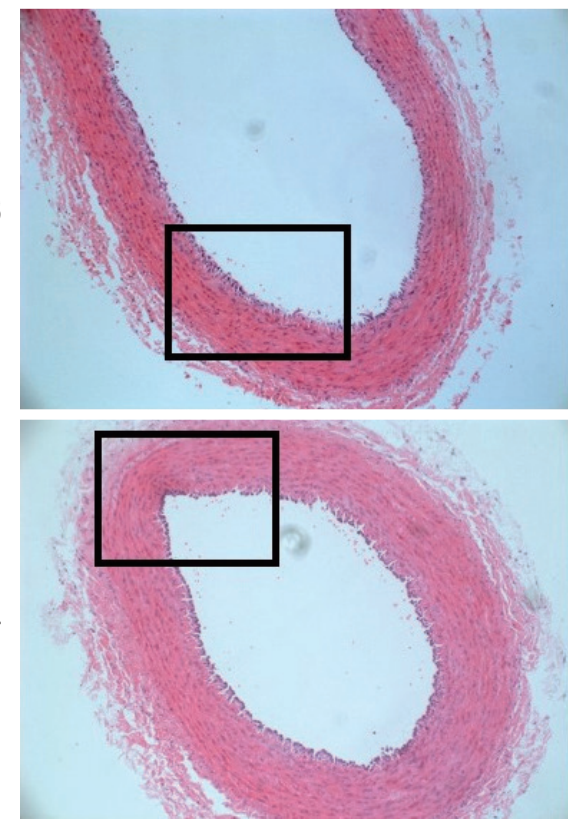
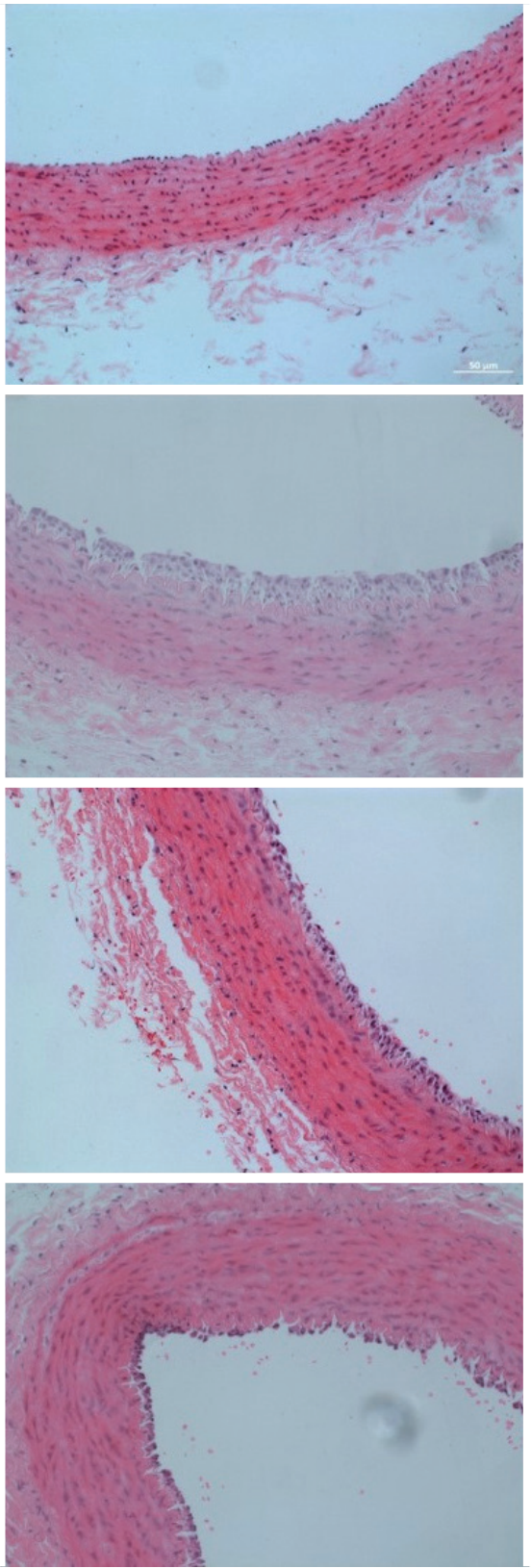
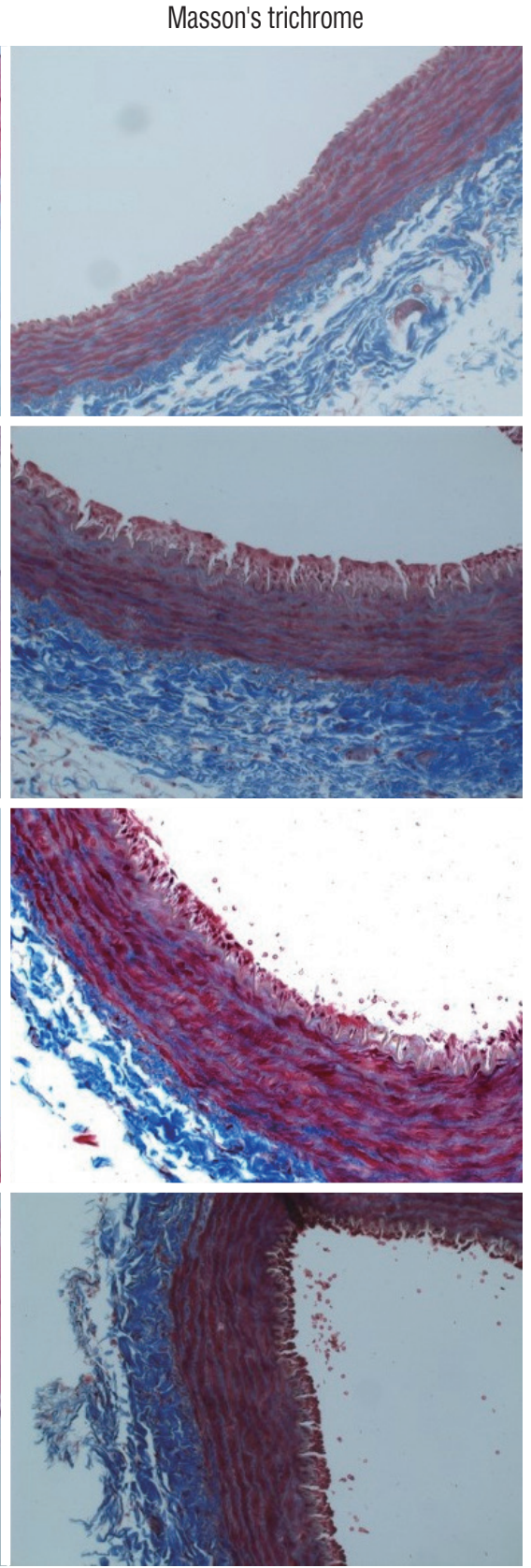

Fig. 3. Microscopic view of the H\&E and Masson's trichrome stained tissues. The tunica intima of the negative control group (group 1) shows a single thin layer of endothelial cells. Marked thickening of tunica intima can be observed in the positive control group (group 2). In the statin-administered groups 3 and 4, a significant decrease in intimal thickening can be observed (magnification $\times 20$ on 1st column, magnification $\times 200$ on 2 nd and 3rd columns). H\&E : hematoxylin and eosin, G1 : group 1, G2 : group 2, G3 : group 3, G4 : group 4.

validating the irritating effects of MT on the vessel wall, and these injury-mediated vessel wall changes (intimal thickening) could be reduced by statin administration (groups 3 and 4 ).

\section{Stent retriever and vessel wall injury}

Despite the remarkable improvement in the complete revas- cularization rate of stent retrievers used for AIS treatment, a difference between the post-procedural radiological result and clinical outcome was observed, causing great concern. One of the possible factors for this was delayed arterial re-occlusion after MT. Given that capturing and retrieving the clots by deploying the stent in the vessel and extracting it in the expand- 
Table 2. Histomorphological analysis of the intimal thickness in each group

\begin{tabular}{|c|c|c|c|c|c|c|}
\hline & Group $1(n=4)$ & Group $2(n=8)$ & Group $3(n=8)$ & Group $4(n=8)$ & $\mathrm{F}^{*}$ & $\operatorname{Sig} \cdot(p)^{*}$ \\
\hline \multicolumn{7}{|l|}{ Intimal thickness $(\mu \mathrm{m})$} \\
\hline$H \& E$ & $3.74 \pm 0.62^{\mathrm{a}}$ & $48.04 \pm 12.28^{d}$ & $30.88 \pm 10.41^{c}$ & $15.86 \pm 3.19^{b}$ & 28.885 & 0.001 \\
\hline Masson's trichrome & $5.84 \pm 0.63^{\mathrm{a}}$ & $42.72 \pm 8.82^{d}$ & $28.36 \pm 9.86^{c}$ & $14.93 \pm 5.75^{b}$ & 26.754 & 0.001 \\
\hline \multicolumn{7}{|c|}{ Immunohistochemistry (area\%) } \\
\hline TNF- $\alpha$ & $10.55 \pm 3.63^{\mathrm{a}}$ & $21.75 \pm 6.78^{c}$ & $15.55 \pm 3.44^{\mathrm{a}, \mathrm{b}}$ & $18.46 \pm 3.44^{b, c}$ & 5.703 & 0.004 \\
\hline CD11b & $11.15 \pm 4.95^{\mathrm{a}}$ & $19.64 \pm 5.19^{b}$ & $14.84 \pm 3.63^{\mathrm{a}, \mathrm{b}}$ & $14.35 \pm 5.27^{\mathrm{a}, \mathrm{b}}$ & 3.303 & 0.037 \\
\hline CD163 & $14.25 \pm 1.28$ & $24.54 \pm 8.59$ & $17.65 \pm 5.86$ & $17.47 \pm 6.62$ & 2.758 & 0.064 \\
\hline
\end{tabular}

Values are presented as mean \pm standard deviation. Post-hoc was performed using Duncan's method $(a<b<c<d)$. ${ }^{*}$ One-way analysis of variance (ANOVA) for three or more groups was performed. H\&E : hematoxylin and eosin, TNF : tumor necrosis factor, CD : cluster of differentiation
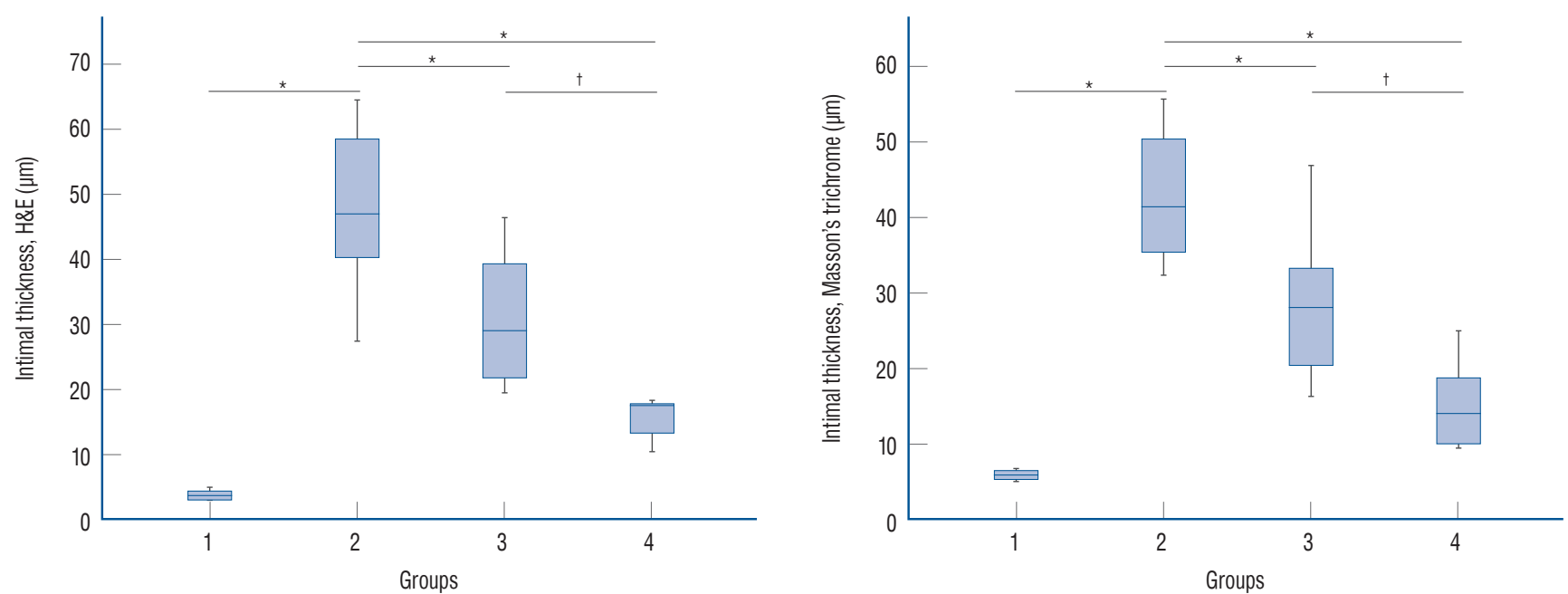

Fig. 4. Histomorphological analysis of the intimal thickness in the hematoxylin and eosin (H\&E) and Masson's trichrome stained tissues. Marked intimal thickening was observed in the groups for which endovascular mechanical thrombectomy was performed. Compared to the positive control group (group 2), the statin-administered groups (groups 3 and 4) showed decreased intimal thickness. The statistical analysis was performed using one-way analysis of variance (ANOVA) for three or more groups. Post-hoc was performed using Duncan's method. ${ }^{*} p<0.05$ vs. the positive control group. ${ }^{\dagger} p<0.05$ between the statin-administered groups (before and after endovascular mechanical thrombectomy).

ed state might damage the inner vessel wall, subsequent changes in the vessel wall were expected to occur, affecting the patency of the re-perfused vessel. One autopsy report showed vascular and parenchymal abnormalities in five patients who expired after $\mathrm{MT}^{39}$. Although a first generation retrieving stent was used for MT, one of the causes of death was vessel occlusion due to a subintimal dissection. Accordingly, clinical analysis of the vessel wall injury was reported. Post-procedural vasospasm and de novo stenosis and occlusion after MT using a second generation retrieving stent have been confirmed to occur in $26.0 \%$ and $3.4 \%$ of the patients, respectively ${ }^{20)}$. In the meantime, several animal studies also demonstrated vessel wall injury associated with the use of stent retrievers ${ }^{2,727,28)}$.
These studies proved that pulling a fully expanded stent denudated the endothelium due to the abrasive force of the expansile stent on the vessel wall. To study the pathomechanism of re-stenosis after vessel wall injury, Perren et al. ${ }^{31)}$ gathered previous reports on animal model-based pathophysiology of this phenomenon and summarized them. They showed that once moderate shear or stress forces were exerted on the vessel wall, the tunica intima was denuded within 48 hours, resulting in platelet and monocyte accumulation at the implicated site. Between days 7 and 14, myointimal cells covered the intima, forming several thick layers at some areas. This is called myointimal hyperplasia. Thereafter, histological evolution depended on the amount of shear force applied to the vessel wall. 


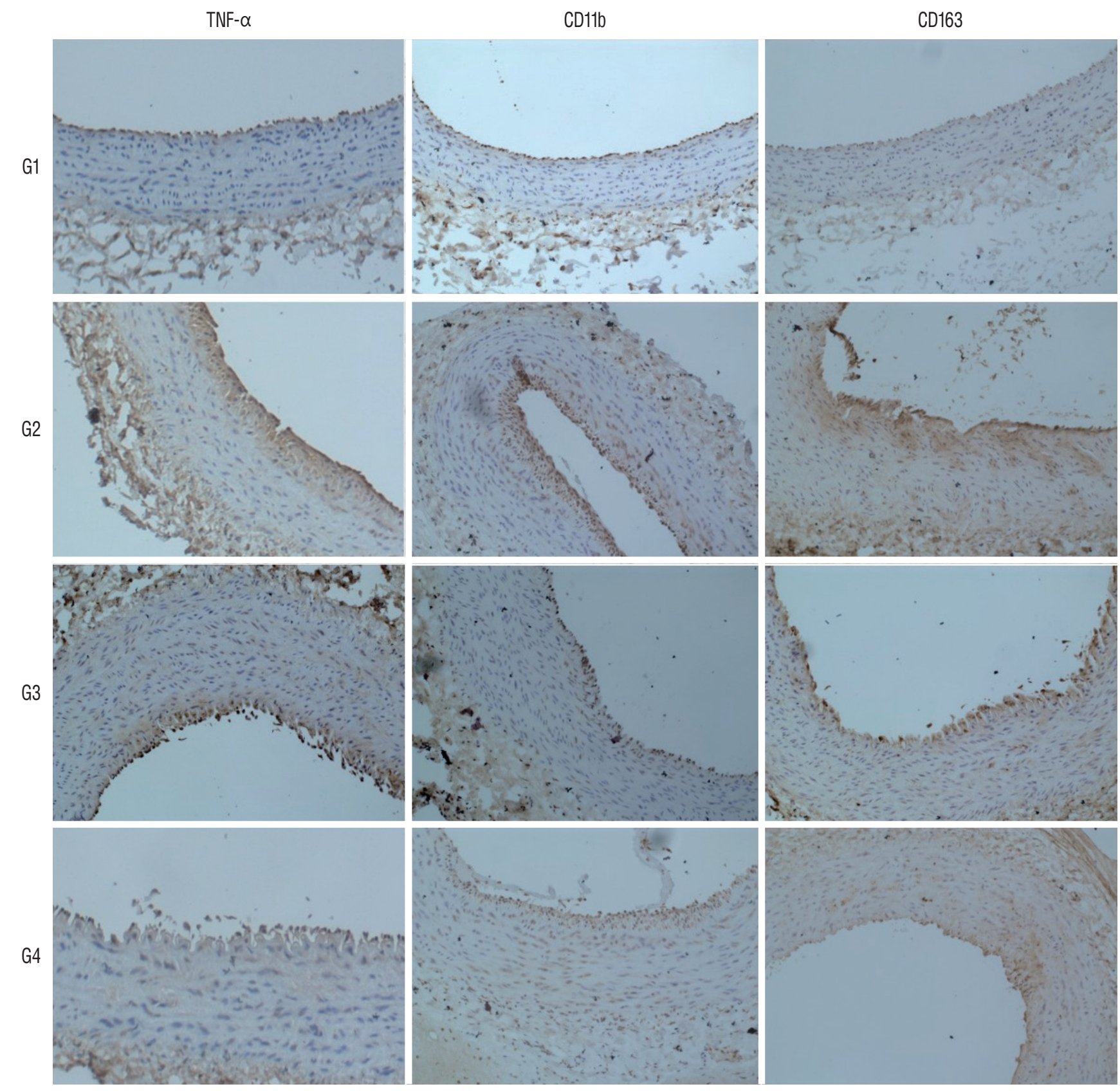

Fig. 5. Microscopic view of the immunohistochemically stained tissues. The stained percentage areas were markedly increased in the positive control group (group 2), compared to in the negative control group (group 1). Statin administration reduced the observed percentage areas of all immunological antibodies (magnification $\times 200$ for all samples). TNF- $\alpha$ : tumor necrosis factor- $\alpha, C D$ : cluster of differentiation, G1 : group 1, G2 : group 2 , G3 : group 3, G4 : group 4.

For stress forces around $120 \mathrm{~Pa}$, complete intimal recovery was expected after 4 weeks. In contrast, shear forces over $200 \mathrm{~Pa}$ fractured the internal elastic lamellae situated in the outermost part of the intima, leading to persistent myointimal hyperplasia. Likewise, Arai et al. ${ }^{2)}$ performed a study using an animal model in which stent retrievers of two different sizes
(Solitare FR $4 \mathrm{~mm}$ vs. Solitare FR $6 \mathrm{~mm}$ ) were used to compare the extent of vessel wall injury. The tendency to develop intimal thickening was more evident when Solitare FR $6 \mathrm{~mm}$ was used. Based on this finding, they postulated that the extent of intimal thickening was strongly correlated with the radial force of the stent. To date, it has been elucidated that stress 

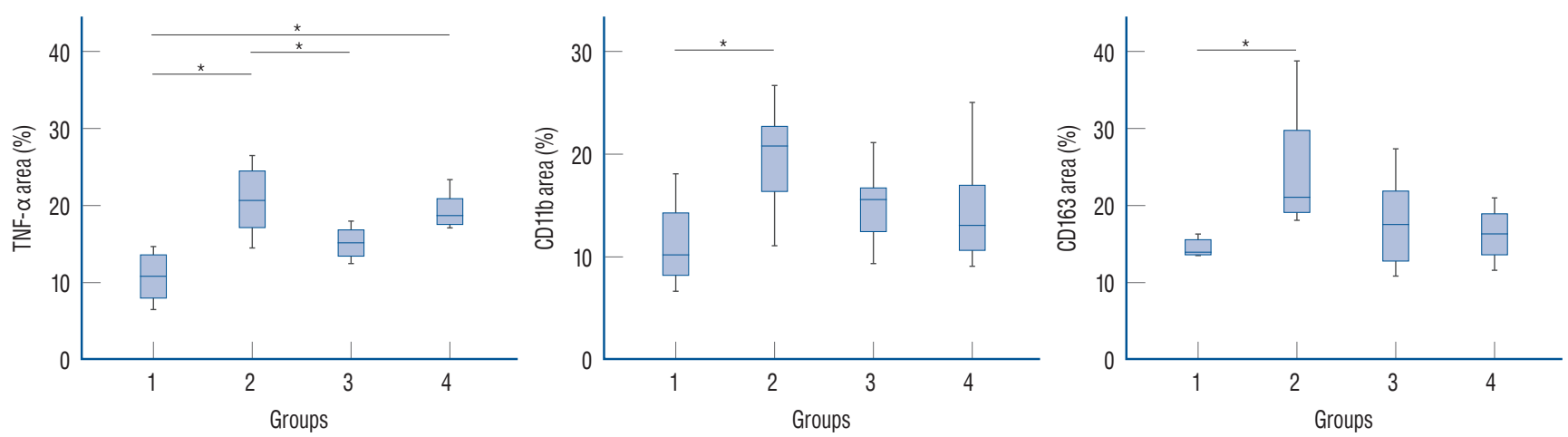

Fig. 6. Immunohistochemical analysis of the inflammatory markers. The percentage area of all immunological antibodies significantly increased in positive control group (group 2), compared to in the negative control group (group 1). In group 3, the percentage area of TNF- $\alpha$ staining was significantly reduced $(p<0.05)$, compared to the positive control group (group 2), but significant differences were not observed for CD11b and CD163 staining. In group 4, no significant differences were observed for TNF- $\alpha, C D 11 b$, and CD163 staining $(p \geq 0.05)$. The statistical analysis was performed using one-way analysis of variance (ANOVA) for three or more groups. ${ }^{*} p<0.05$ vs. positive control group. TNF- $\alpha$ : tumor necrosis factor- $\alpha, C D$ : cluster of differentiation.

forces applied to a vessel wall can induce a myointimal reaction, leading to transient or permanent hyperplasia. Subsequently, myointimal hyperplasia may regress or progress into severe stenosis depending on the degree of radial force exerted on the vessel wall ${ }^{29,37)}$.

In line with previous literature, our study showed a significant increase in the intimal thickening in the positive control group $(48.04 \pm 12.28 \mu \mathrm{m}$ of $\mathrm{H} \& \mathrm{E}$ and $42.72 \pm 8.82 \mu \mathrm{m}$ of Masson's trichrome), compared to in the negative control group (3.74 $\pm 0.62 \mu \mathrm{m}$ of H\&E stain and $5.84 \pm 0.63 \mu \mathrm{m}$ of Masson's trichrome). Additionally, intimal thickening was confirmed to be associated with the inflammatory reactions elicited by direct mechanical injury of the vessel wall. TNF- $\alpha$, CD11b, and CD163, the immunohistochemical stains of macrophage markers used for assessing the inflammatory reactions, were markedly increased in the positive control group (group 2). TNF- $\alpha$, which was initially described as a circulating factor associated with tumor necrosis, is a key regulator of inflammatory response. It is mainly secreted by activated macrophages, and it sends signals to vascular endothelial cells, exerting pro-inflammatory changes, such as leukocyte adhesion, transendothelial migration, and vascular leak, and ultimately promoting thrombosis and plaque formation ${ }^{6}$. The significant increase in TNF- $\alpha$ in the positive control showed that MT-induced inner vessel wall injury initiated the inflammatory responses, sequentially leading to intimal thickening. Likewise, CD11b and CD163, which are markers for macrophages observed in advanced plaques, were consistently increased in the positive control group, thus supporting the evidence on the presence of an immune response against the inner vessel wall injury. Although it failed to reduce all immunohistochemical variables in the statin-administered groups (groups 3 and 4) significantly, TNF- $\alpha$ significantly reduced these variables in group 3. Other variables in groups 3 and 4 , compared to those in the positive control group, were also substantially reduced, but failed to show statistically significant differences. Since there have been reports that statins exert prohibitory effects on atherosclerotic plaque formation by reducing macrophage content ${ }^{19,24)}$, the difference in the window period during which the rabbit carotid arteries were harvested between this study (1 week) and previous literature (2 or more weeks) was possibly the reason why our immunohistochemical results failed to show significance.

\section{The role of statins in vessel wall protection}

Statins, identified as fungal metabolites, are HMG-CoA reductase inhibitors, and have been on the market since $1987^{26,35,38)}$. Since HMG-CoA reductase is the rate-limiting enzyme for cholesterol biosynthesis in the liver, its inhibition results in decreased cholesterol production and LDL receptor up-regulation ${ }^{17)}$. Large RCTs have proven that statins effectively prevent coronary heart disease by exerting either LDLcholesterol-dependant or LDL-cholesterol-independent effects $^{25}$. The LDL-cholesterol-independent effect, also known as the 'pleiotropic' effect, showed that the overall benefits observed due to statin treatment were more significant than those expected due to lipid level decrease. The pleiotropic effect of statins is known to improve endothelial function, en- 
hance atherosclerotic plaque stability, decrease oxidative stress and inflammation, and inhibit the thrombogenic response $\mathrm{e}^{25}$. Specifically, the pleiotropic effect on enhancing endothelial function is profoundly associated with the bioavailability of endothelial-derived nitric oxide (NO). Endothelial-derived $\mathrm{NO}$ is essential for vasodilation, platelet aggregation, vascular smooth muscle proliferation, and endothelial-leukocyte interactions $^{14)}$. By exerting this pleiotropic effect, statins can increase endothelial NO production by up-regulating endothelial NO synthase ${ }^{21,22)}$. Besides, statins can decrease plateletderived growth factor (PDGF)-induced DNA synthesis in vascular smooth muscle cells, leading to reduced cellular proliferation, leukocyte accumulation, and PDGF receptor phosphorylation and thus decreasing intimal thickening ${ }^{9,23)}$.

Accordingly, the pleiotropic effect of statins on the endothelium and vascular smooth muscle cell played an important role in this study. On comparing the changes in the intimal thickness after MT, we observed that the statin-administered group showed a lesser increase in intimal thickness than the control groups, showing statistically significant differences between group 2 and groups 3 and 4 (both were $p=0.001$ ).

Interestingly, the increase in intimal thickness was far lesser in group 4 than in group 3. The measured intimal thickness in group 4 was almost half of that in group 3 and one-third of that in group 2. We believe that this phenomenon was associated with the pharmacokinetic nature of statins. Since the plasma half-life of atorvastatin is 14 hours, the protective effects on the vessel after statin discontinuation will decrease abruptly. Therefore, the efficacy of statins was probably lesser in group 3 (statin pre-administered) than in group 4 (statin post-administered). Substantially, the observed findings of the changes in the intimal thickness and processed statins in group 4 were far different from those in group 3 (see the boxplots of Fig. 4). Meanwhile, this finding could be another evidence for the direct effect of statins on vessel wall protection (pleiotropic effect), and not the adjunct plasma lipid-lowering effect of statins. Nevertheless, the exact reason should be investigated in future studies.

In the present study, we used rabbit carotid arteries to investigate the vascular damage caused due to endovascular MT using stent retrievers and identify the protective effects of statins. The MT-induced endothelial injury was confirmed to occur as not only an increase in intimal thickening but also an inflammatory reaction. The increase in intimal thickening was reduced in the statin-administered groups, among which the statin post-administered group showed increased intimal thickness that was one-third of that in the non-statin-administered group and half of that in the statin pre-administered group. The efficacy of the vascular protective effects of statins was higher when the statin was given after MT than when it was given before MT.

\section{Limitations}

Although this study provided insights into the protective effects of statins towards MT-induced vessel wall injury, it also showed several limitations. Because our research focused on the early protective effect of statin towards injured vessel after MT, we were unable to show the long-term changes in the intimal layer after MT-induced vessel wall injury with or without statin administration. As the first step to confirm the protective effects of statins on the vessel wall, this study could be a cornerstone for resolving the questions on the diverse clinical outcomes despite successful recanalization with MT. The serum lipid and inflammatory factor levels were not included in this study. Since statins exert both LDL-cholesterol-dependant and LDL-cholesterol-independent effects for vascular protection, measuring serum lipid levels may provide convincing evidence of the pleiotropic effect of statins.

Nevertheless, taking into account the different results of groups 3 and 4, we could observe the pleiotropic effect of statins indirectly. Lastly, a relatively small animal population was used in this study to establish the whole hypothesis of MT-induced vascular injury and the protective effects of statins towards it. A well-planned additional study with a larger sample size will add weight to the conclusion of our hypothesis. And, another experimental design using transgenic animal model of lipid metabolic disorder and another immunohistochemical stain will be considered to prove the relationship between vessel injury after MT and healing mechanism of statin administration.

\section{CONCLUSION}

Endovascular MT plays an important role in AIS, which is more common in the elderly. As the aging population increases, the use of stent retrieving devices will rise steeply. Although the stent retrieving device has proven to be effective in 
recanalization of large occluded vessels, vascular wall injury during blood clot extraction is a cause of great concern. In this study, we showed that MT induced endothelial changes by measuring the changes in the intimal thickness. Furthermore, we presented that statin administration before and after MT exerted protective effects towards vessel wall injury. The efficacy of statins was greater post-administration than pre-administration. Thus, including statin administration in routine prescriptions in the peri-procedural period is worth considering.

\section{CONFLICTS OF INTEREST}

No potential conflict of interest relevant to this article was reported.

\section{INFORMED CONSENT}

This type of study does not require informed consent.

\section{AUTHOR CONTRIBUTIONS}

\author{
Conceptualization : HSS \\ Data curation : HSS \\ Formal analysis : HSS \\ Funding acquisition : HSS \\ Methodology : IO \\ Project administration : SHL \\ Visualization : HSS \\ Writing - original draft : HSS, SHL \\ Writing - review \& editing : IO
}

\section{ORCID}

Seung Hwan Lee https://orcid.org/0000-0001-8043-632X

Hee Sup Shin Thtps://orcid.org/0000-0002-5286-8448

Inho $\mathrm{Oh}$

\section{- Acknowledgements}

This work was supported by a grant from Kyung Hee University in 2018 (KHU-20180940).

We would like to thank Jong Un Moon for the technical support in animal experiments.

\section{References}

1. Alexandrov AV, Molina CA, Grotta JC, Garami Z, Ford SR, Alvarez-Sabin J, et al. : Ultrasound-enhanced systemic thrombolysis for acute ischemic stroke. N Engl J Med 351 : 2170-2178, 2004

2. Arai $D$, Ishii $A$, Chihara $H$, Ikeda $H$, Miyamoto $S$ : Histological examination of vascular damage caused by stent retriever thrombectomy devices. J Neurointerv Surg 8 : 992-995, 2016

3. Auerbach BJ, Krause BR, Bisgaier CL, Newton RS : Comparative effects of HMG-CoA reductase inhibitors on apo B production in the casein-fed rabbit: atorvastatin versus lovastatin. Atherosclerosis 115 : 173-180, 1995

4. Berkhemer OA, Fransen PS, Beumer D, van den Berg LA, Lingsma HF, Yoo AJ, et al. : A randomized trial of intraarterial treatment for acute ischemic stroke. N Engl J Med 372 : 11-20, 2015

5. Bhatia R, Hill MD, Shobha N, Menon B, Bal S, Kochar P, et al. : Low rates of acute recanalization with intravenous recombinant tissue plasminogen activator in ischemic stroke: real-world experience and a call for action. Stroke 41 : 2254-2258, 2010

6. Bradley JR : TNF-mediated inflammatory disease. J Pathol 214 : 149160,2008

7. Brekenfeld C, Schroth G, El-Koussy M, Nedeltchev K, Reinert M, Slotboom J, et al. : Mechanical thromboembolectomy for acute ischemic stroke: comparison of the catch thrombectomy device and the Merci Retriever in vivo. Stroke 39 : 1213-1219, 2008

8. Campbell BC, Mitchell PJ, Kleinig TJ, Dewey HM, Churilov L, Yassi N, et al. : Endovascular therapy for ischemic stroke with perfusion-imaging selection. N Engl J Med 372 : 1009-1018, 2015

9. Chen Z, Fukutomi T, Zago AC, Ehlers R, Detmers PA, Wright SD, et al. : Simvastatin reduces neointimal thickening in low-density lipoprotein receptor-deficient mice after experimental angioplasty without changing plasma lipids. Circulation 106 : 20-23, 2002

10. del Zoppo GJ, Poeck K, Pessin MS, Wolpert SM, Furlan AJ, Ferbert A, et al. : Recombinant tissue plasminogen activator in acute thrombotic and embolic stroke. Ann Neurol 32 : 78-86, 1992

11. Faul F, Erdfelder E, Buchner A, Lang AG : Statistical power analyses using $G^{*}$ Power 3.1: tests for correlation and regression analyses. Behav Res Methods 41 : 1149-1160, 2009

12. Faul F, Erdfelder E, Lang AG, Buchner A : $G^{*}$ Power 3: a flexible statistical power analysis program for the social, behavioral, and biomedical sciences. Behav Res Methods 39 : 175-191, 2007 
13. Gascou G, Lobotesis K, Machi P, Maldonado I, Vendrell JF, Riquelme $C$, et al. : Stent retrievers in acute ischemic stroke: complications and failures during the perioperative period. AJNR Am J Neuroradiol 35 : 734-740, 2014

14. Gauthier TW, Scalia R, Murohara T, Guo JP, Lefer AM : Nitric oxide protects against leukocyte-endothelium interactions in the early stages of hypercholesterolemia. Arterioscler Thromb Vasc Biol 15 : 16521659, 1995

15. Goyal M, Demchuk AM, Menon BK, Eesa M, Rempel JL, Thornton J, et al. : Randomized assessment of rapid endovascular treatment of ischemic stroke. N Engl J Med 372 : 1019-1030, 2015

16. Haussen DC, Rebello LC, Nogueira RG : Optimizating clot retrieval in acute stroke: the push and fluff technique for closed-cell stentrievers. Stroke 46 : 2838-2842, 2015

17. Istvan ES, Deisenhofer J : Structural mechanism for statin inhibition of HMG-CoA reductase. Science 292 : 1160-1164, 2001

18. Jovin TG, Chamorro A, Cobo E, de Miquel MA, Molina CA, Rovira A, et al. : Thrombectomy within 8 hours after symptom onset in ischemic stroke. N Engl J Med 372 : 2296-2306, 2015

19. Kato M, Sada T, Mizuno M, Kitayama K, Inaba T, Koike H : Effect of combined treatment with an angiotensin II receptor antagonist and an HMG-CoA reductase inhibitor on atherosclerosis in genetically hyperlipidemic rabbits. J Cardiovasc Pharmacol 46 : 556-562, 2005

20. Kurre W, Pérez MA, Horvath $D$, Schmid E, Bäzner $H$, Henkes $H$ : Does mechanical thrombectomy in acute embolic stroke have long-term side effects on intracranial vessels? An angiographic follow-up study. Cardiovasc Intervent Radiol 36 : 629-636, 2013

21. Laufs U, La Fata V, Plutzky J, Liao JK : Upregulation of endothelial nitric oxide synthase by HMG CoA reductase inhibitors. Circulation 97 : 1129-1135, 1998

22. Laufs U, Liao JK : Post-transcriptional regulation of endothelial nitric oxide synthase mRNA stability by Rho GTPase. J Biol Chem 273 : 2426624271, 1998

23. Laufs U, Marra D, Node K, Liao JK : 3-Hydroxy-3-methylglutaryl-CoA reductase inhibitors attenuate vascular smooth muscle proliferation by preventing rho GTPase-induced down-regulation of p27(Kip1). J Biol Chem 274 : 21926-21931, 1999

24. Lee SG, Lee SJ, Thuy NVP, Kim JS, Lee JJ, Lee OH, et al. : Synergistic protective effects of a statin and an angiotensin receptor blocker for initiation and progression of atherosclerosis. PLoS One 14 : e0215604, 2019

25. Liao JK, Laufs U : Pleiotropic effects of statins. Annu Rev Pharmacol Toxicol 45 : 89-118, 2005

26. McTaggart $\mathrm{F}$ : Comparative pharmacology of rosuvastatin. Atheroscler Suppl 4 : 9-14, 2003

27. Mordasini P, Hiller M, Brekenfeld C, Schroth G, Fischer U, Slotboom J, et al. : In vivo evaluation of the Phenox CRC mechanical thrombectomy device in a swine model of acute vessel occlusion. AJNR Am J Neuroradiol 31 : 972-978, 2010

28. Mordasini P, Frabetti N, Gralla J, Schroth G, Fischer U, Arnold M, et al. : In vivo evaluation of the first dedicated combined flow-restoration and mechanical thrombectomy device in a swine model of acute vessel occlusion. AJNR Am J Neuroradiol 32 : 294-300, 2011

29. Neville RF, Sidawy AN : Myointimal hyperplasia: basic science and clinical considerations. Semin Vasc Surg 11 : 142-148, 1998

30. Pecoraro V, Moja L, Dall'Olmo L, Cappellini G, Garattini S : Most appropriate animal models to study the efficacy of statins: a systematic review. Eur J Clin Invest 44 : 848-871, 2014

31. Perren F, Kargiotis O, Pignat JM, Pereira VM : Hemodynamic changes may indicate vessel wall injury after stent retrieval thrombectomy for acute stroke. J Neuroimaging 28 : 412-415, 2018

32. Powers WJ, Rabinstein AA, Ackerson T, Adeoye OM, Bambakidis NC, Becker $\mathrm{K}$, et al. : Guidelines for the early management of patients with acute ischemic stroke: 2019 update to the 2018 guidelines for the early management of acute ischemic stroke: a guideline for healthcare professionals from the American Heart Association/American Stroke Association. Stroke 50 : e344-e418, 2019

33. Ribo M, Alvarez-Sabín J, Montaner J, Romero F, Delgado P, Rubiera M, et al. : Temporal profile of recanalization after intravenous tissue plasminogen activator: selecting patients for rescue reperfusion techniques. Stroke 37 : 1000-1004, 2006

34. Saver JL, Goyal M, Bonafe A, Diener HC, Levy El, Pereira VM, et al. : Stent-retriever thrombectomy after intravenous t-PA vs. t-PA alone in stroke. N Engl J Med 372 : 2285-2295, 2015

35. Sirtori $C R$ : The pharmacology of statins. Pharmacol Res $88: 3-11$, 2014

36. Smith WS : Safety of mechanical thrombectomy and intravenous tissue plasminogen activator in acute ischemic stroke. Results of the multi Mechanical Embolus Removal in Cerebral Ischemia (MERCI) trial, part I.

AJNR Am J Neuroradiol 27 : 1177-1182, 2006

37. Sterpetti AV, Cucina A, Lepidi S, Randone B, Stipa F, Aromatario C, et al. : Progression and regression of myointimal hyperplasia in experimental vein grafts depends on platelet-derived growth factor and basic fibroblastic growth factor production. J Vasc Surg 23 : 568-575, 1996

38. Stone NJ, Robinson JG, Lichtenstein AH, Bairey Merz CN, Blum CB, Eckel RH, et al. : 2013 ACC/AHA guideline on the treatment of blood cholesterol to reduce atherosclerotic cardiovascular risk in adults: a report of the American College of Cardiology/American Heart Association task force on practice guidelines. Circulation 129 : S1-S45, 2014

39. Yin NS, Benavides S, Starkman S, Liebeskind DS, Saver JA, Salamon N, et al. : Autopsy findings after intracranial thrombectomy for acute ischemic stroke: a clinicopathologic study of 5 patients. Stroke 41 : 938947, 2010 